\title{
On the univalent, bounded, non-vanishing and symmetric functions in the unit disk
}

\author{
by J. ŚLADKOWSKA (Gliwice)
}

\begin{abstract}
The paper is devoted to a class of functions analytic, univalent, bounded and non-vanishing in the unit disk and in addition, symmetric with respect to the real axis. Variational formulas are derived and, as applications, estimates are given of the first and second coefficients in the considered class of functions.
\end{abstract}

1. Introduction. Let $\mathcal{B}_{0}^{R}(b), 0<|b|<1$, denote the class of all functions that are analytic, univalent in the unit disk $U$ and satisfy the conditions

$$
f(U) \subset U, \quad f(0)=b, \quad 0 \notin f(U), \quad \operatorname{Im} f^{(n)}(0)=0, \quad n=0,1, \ldots
$$

$\mathcal{B}_{0}^{R}(b)$ is obviously a normal family but not compact in the topology of uniform convergence on compact subsets. However, it becomes compact by addition of the function $f_{b}$ which is identically equal to $b$.

The main aim of the present paper is to obtain variations in $\mathcal{B}_{0}^{R}(b)$ that would be rich enough to derive the estimates of some functionals in this class. We shall use the techniques developed by Hummel and Schiffer in [3].

$\mathcal{B}_{0}^{R}(b)$ is a subclass of the class $\mathcal{B}_{0}(b)$ of all functions $f$ analytic and univalent in $U$ with $f(U) \subset U, f(0)=b$ and $0 \notin f(U)$. Variations in this class were constructed by Hummel and Pinchuk in [2].

2. Elementary variational formulas. We can obtain useful varied functions by transformations in the $z$-plane. Let $\omega(z)$ be analytic and univalent in $U, \omega(U) \subset U, \omega(0)=0, \operatorname{Im} \omega^{(n)}(0)=0, n=1,2, \ldots$ Then $f \circ \omega \in \mathcal{B}_{0}^{R}(b)$. In particular, putting $\omega(z)=(1-\varepsilon) z, 0<\varepsilon<1$, we have the varied functions in $\mathcal{B}_{0}^{R}(b)$

$$
f^{*}(z)=f((1-\varepsilon) z)=f(z)-\varepsilon z f^{\prime}(z)+o(\varepsilon) .
$$

1991 Mathematics Subject Classification: Primary 30C45.

Key words and phrases: univalent function, variational method, Schiffer type equation. 
To obtain the slit variation in $\mathcal{B}_{0}^{R}(b)$, we consider the differential equation

$$
\frac{\partial w}{\partial t}=-\omega p(\omega)
$$

where

$$
p(\omega)=\frac{1}{2}\left(\frac{1+e^{-i \alpha} \omega}{1-e^{-i \alpha} \omega}+\frac{1+e^{i \alpha} \omega}{1-e^{i \alpha} \omega}\right), \quad \alpha \in \mathbb{R} .
$$

Then, by Theorem 6.3 of [4], p. 160, the equation (2) has for each $z \in U$ a unique solution $\omega(z, t), 0 \leq t<\infty$, with the initial condition $\omega(z, 0)=z$, such that the function $\omega(z, t)$ is absolutely continuous in $0 \leq t<\infty$ for each $z \in U$ and univalent in $z \in U$ for each $0 \leq t<\infty$. Furthermore, $\omega(0, t)=$ $0,|\omega(z, t)|<1$ and, since $p(\bar{\omega})=\overline{p(\omega)}$, we also have $\omega(\bar{z}, t)=\overline{\omega(z, t)}$. Consequently, $\omega(z, t)$ as an analytic function of $z$ has real coefficients. Hence, if $f$ belongs to $\mathcal{B}_{0}^{R}(b)$, then $f^{*}(z)=f(\omega(z, \varepsilon)), 0<\varepsilon<\infty$, also belongs to $\mathcal{B}_{0}^{R}(b)$, having the varied formula

$$
f^{*}(z)=f(z)-\frac{1}{2} \varepsilon z f^{\prime}(z)\left(\frac{1+e^{-i \alpha} z}{1-e^{-i \alpha} z}+\frac{1+e^{i \alpha} z}{1-e^{i \alpha} z}\right)+o(\varepsilon) .
$$

3. Hummel-Schiffer variational formulas. Let $D=f(U)$, where $f \in \mathcal{B}_{0}^{R}(b)$, and let $w_{0} \notin \partial f(U)$. Let

$$
w^{*}(w)=w \exp \{\varepsilon \Phi(w)\}, \quad \varepsilon>0,
$$

where

(5) $\Phi(w)=e^{i \alpha} \frac{w+w_{0}}{w-w_{0}}+e^{-i \alpha} \frac{w+\bar{w}_{0}}{w-\bar{w}_{0}}-e^{-i \alpha} \frac{1+\bar{w}_{0} w}{1-\bar{w}_{0} w}-e^{i \alpha} \frac{1+w_{0} w}{1-w_{0} w}$,

$\alpha \in \mathbb{R}, \varepsilon>0$. It is evident that $w^{*}(w)$ is an analytic function in some domain $\Delta$ containing the boundary $\partial D, w^{*}(w) \neq 0$ for $w \in D(0 \notin D)$, $\left|w^{*}(w)\right|=1$ for $w \in \partial U$, and if $f \in \mathcal{B}_{0}^{R}(b)$, then $w^{*}(\bar{w})=\overline{w^{*}(w)}$. It can be proved quite easily that $w^{*}(w)$ is univalent in $\Delta$ for $\varepsilon$ sufficiently small and for all $\alpha \in \mathbb{R}$. It follows from the above that $w^{*}(w)$ maps the boundary $\partial D$ onto the boundary of a new domain $D^{*}$ such that $D^{*} \subset U, 0 \notin D^{*}, b \in D^{*}$, and $D^{*}$ is symmetric about the real axis.

Basing now on Goluzin's method of constructing variations of functions of the class $S([1]$, p. 98), we obtain a varied function in the form

$$
\begin{aligned}
f^{*}(z)= & f(z)+\varepsilon\left(e^{i \alpha} f(z) \frac{f(z)+w_{0}}{f(z)-w_{0}}+e^{-i \alpha} f(z) \frac{f(z)+\bar{w}_{0}}{f(z)-\bar{w}_{0}}\right. \\
& -e^{-i \alpha} f(z) \frac{1+\bar{w}_{0} f(z)}{1-\bar{w}_{0} f(z)}-e^{i \alpha} f(z) \frac{1+w_{0} f(z)}{1-w_{0} f(z)} \\
& -\left(1-z^{2}\right) f^{\prime}(z)\left(e^{i \alpha} \frac{b\left(b+w_{0}\right)}{b_{1}\left(b-w_{0}\right)}+e^{-i \alpha} \frac{b\left(b+\bar{w}_{0}\right)}{b_{1}\left(b-\bar{w}_{0}\right)}\right. \\
& \left.\left.-e^{-i \alpha} \frac{b\left(1+\bar{w}_{0} b\right)}{b_{1}\left(1-\bar{w}_{0} b\right)}-e^{i \alpha} \frac{b\left(1+w_{0} b\right)}{b_{1}\left(1-w_{0} b\right)}\right)\right)+o(\varepsilon),
\end{aligned}
$$


where $w_{0} \notin \overline{f(U)}$, and

$$
\begin{aligned}
f^{*}(z)= & f(z)+\varepsilon\left(e^{i \alpha} f(z) \frac{f(z)+f(\zeta)}{f(z)-f(\zeta)}+e^{-i \alpha} f(z) \frac{f(z)+\overline{f(\zeta)}}{f(z)-\overline{f(\zeta)}}\right. \\
& -e^{-i \alpha} f(z) \frac{1+\overline{f(\zeta)} f(z)}{1-\overline{f(\zeta)} f(z)}-e^{i \alpha} f(z) \frac{1+f(\zeta) f(z)}{1-f(\zeta) f(z)} \\
& -\left(1-z^{2}\right) f^{\prime}(z)\left(e^{i \alpha} \frac{b(b+f(\zeta))}{b_{1}(b-f(\zeta))}+e^{-i \alpha} \frac{b(b+\overline{f(\zeta)})}{b_{1}(b-\overline{f(\zeta)})}\right. \\
& \left.-e^{-i \alpha} \frac{b(1+\overline{f(\zeta)} b)}{b_{1}(1-\overline{f(\zeta)} b)}-e^{i \alpha} \frac{b(1+f(\zeta) b)}{b_{1}(1-f(\zeta) b)}\right) \\
& -z f^{\prime}(z) e^{i \alpha} \frac{2 f^{2}(\zeta)}{\zeta f^{\prime 2}(\zeta)} \frac{1}{z-\zeta}-z f^{\prime}(z) e^{-i \alpha} \frac{2 \frac{2 f^{2}(\zeta)}{\overline{f^{\prime 2}(\zeta)}} \frac{1}{z-\bar{\zeta}}}{} \\
& \left.+z f^{\prime}(z) e^{-i \alpha} \frac{2 \overline{f^{2}(\zeta)}}{\bar{\zeta} \overline{f^{\prime 2}(\zeta)}} \frac{z}{1-\bar{\zeta} z}+z f^{\prime}(z) e^{i \alpha} \frac{2 f^{2}(\zeta)}{\overline{\zeta f^{\prime 2}(\zeta)}} \frac{z}{1-\zeta z}\right) \\
& +o(\varepsilon), \quad
\end{aligned}
$$

where $\zeta=f^{-1}\left(w_{0}\right), w_{0} \in f(U)$. In all formulas (1), (3), (6), (7), o( $(\varepsilon) / \varepsilon \rightarrow 0$ as $\varepsilon \rightarrow 0$, uniformly on compact subsets of $U$.

4. Schiffer equation. Let $H(U)$ denote as usual the space of all functions analytic in $U$. Let $\Psi$ be a complex, continuous functional on $\mathcal{B}_{0}^{R}(b)$, having a complex Fréchet derivative at $f$. Then there exists a functional $L_{f} \in H^{\prime}(U)$ such that

$$
\Psi\left(f^{*}\right)=\Psi(f)+\varepsilon L_{f}(h)+o(\varepsilon),
$$

where

$$
f^{*}(z)=f(z)+\varepsilon h(z)+o(\varepsilon),
$$

for every function $h \in H(U), \varepsilon \in \mathbb{R}$, with $o(\varepsilon) / \varepsilon \rightarrow 0$ as $\varepsilon \rightarrow 0$, uniformly on compact subsets of $U$.

The set $\mathcal{B}_{0}^{R}(b) \cup\left\{f_{b}\right\}$ is compact, thus the problem of maximizing and minimizing $\operatorname{Re} \Psi$ in this set, if of course $\Psi$ is defined also for $f_{b}$, always has a solution and this solution is in $\mathcal{B}_{0}^{R}(b)$, if we verify that $f_{b}$ is not maximal or minimal.

Suppose now that $f \in \mathcal{B}_{0}^{R}(b)$ is locally maximal for $\operatorname{Re} \Psi$, that is, $\operatorname{Re} \Psi\left(f^{*}\right)$ $\leq \operatorname{Re} \Psi(f)$ for all "nearby" $f^{*} \in \mathcal{B}_{0}^{R}(b)$ ("nearby" in the sense of uniform convergence on compact subsets). Using the varied functions (1), (3), (6), (7) and the formula (8), we can prove (quite analogously to [6]-[8])

THEOREM 1. Let $\Psi$ be a complex-valued functional defined and continuous on $\mathcal{B}_{0}^{R}(b)$, having a complex Fréchet derivative $L_{f}$ as defined in (8), 
where $f \in \mathcal{B}_{0}^{R}(b)$ is locally maximal for $\operatorname{Re} \Psi$. Then $f$ has the following properties.

(i) $w=f(\zeta)$ satisfies the differential equation

$$
\begin{aligned}
& \text { (9) } \frac{\zeta^{2} w^{\prime 2}}{2 w^{2}}\left\{L_{f}\left(f \frac{f+w}{f-w}-f \frac{1+w f}{1-w f}-\left(1-z^{2}\right) f^{\prime}\left(\frac{b(b+w)}{b_{1}(b-w)}-\frac{b(1+b w)}{b_{1}(1-b w)}\right)\right)\right. \\
& \left.+\overline{L_{f}\left(f \frac{f+\bar{w}}{f-\bar{w}}-f \frac{1+\bar{w} f}{1-\bar{w} f}-\left(1-z^{2}\right) f^{\prime}\left(\frac{b(b+\bar{w})}{b_{1}(b-\bar{w})}-\frac{b(1+b \bar{w})}{b_{1}(1-b \bar{w})}\right)\right)}\right\} \\
& =L_{f}\left(z f^{\prime}\left(\frac{\zeta}{z-\zeta}-\frac{z \zeta}{1-z \zeta}\right)\right)+\overline{L_{f}\left(z f^{\prime}\left(\frac{\bar{\zeta}}{z-\bar{\zeta}}-\frac{z \bar{\zeta}}{1-z \bar{\zeta}}\right)\right)}
\end{aligned}
$$

in some annulus $P=\{\zeta: r<|\zeta|<1\}$.

(ii) $\operatorname{Re} L_{f}\left(z f^{\prime}\right) \geq 0$ and

$$
\operatorname{Re} L_{f}\left(\left(1-z^{2}\right) f^{\prime}-\frac{f^{\prime}(0)}{1-b^{2}}\left(1-f^{2}\right)\right)=0 .
$$

(iii) The right-hand side of (9) is analytic in the annulus $P_{1}=\{\zeta: r<$ $|\zeta|<1 / r\}$, real and non-positive on $\partial U$.

(iv) $f$ maps $U$ onto a domain whose boundary is made up of analytic arcs which lie on trajectories of the quadratic differential $A(w) d w^{2} / w^{2}$, where

$$
\begin{aligned}
& A(w)=L_{f}\left(f \frac{f+w}{f-w}-f \frac{1+f w}{1-f w}\right. \\
& \left.-\left(\frac{b(b+w)}{b_{1}(b-w)}-\frac{b(1+b w)}{b_{1}(1-b w)}\right)\left(1-z^{2}\right) f^{\prime}\right) \\
& +\frac{L_{f}\left(f \frac{f+\bar{w}}{f-\bar{w}}-f \frac{1+f \bar{w}}{1-f \bar{w}}-\left(\frac{b(b+\bar{w})}{b_{1}(b-\bar{w})}-\frac{b(1+b \bar{w})}{b_{1}(1-b \bar{w})}\right)\left(1-z^{2}\right) f^{\prime}\right)}{} .
\end{aligned}
$$

(v) If $A(w)$ in (10) is a rational function $\not \equiv$ const, then $U \backslash f(U)$ has no interior points. The point 0 is on the boundary $\partial f(U)$.

Remark. When $f \in \mathcal{B}_{0}^{R}(b)$ is locally minimal for $\operatorname{Re} \Psi$ then in (ii) we have the inequality $\operatorname{Re} L_{f}\left(z f^{\prime}\right) \leq 0$ and in (iii) the right-hand side of (9) is non-negative on $\partial U$.

5. Estimation of $b_{1}=f^{\prime}(0)$ in $\mathcal{B}_{0}^{R}(b)$. We assume that $0<b<1$ and find, by Theorem $1(\mathrm{i})$, (ii), that if $f \in \mathcal{B}_{0}^{R}(b)$ locally maximizes $b_{1}$, then $b_{1}>0$ and $f$ satisfies the differential equation

$$
\frac{\zeta^{2} w^{\prime 2}}{w^{2}} \cdot \frac{P(w)}{(w-b)^{2}(1-b w)^{2}}=-b_{1}^{2},
$$

where $P(w)=A w^{4}+B w^{3}+C w^{2}+D w+E$ and $A, B, C, D, E$ are real. From the symmetry with respect to $\partial U$ of the expression in braces on the 
left-hand side of (9) we conclude that $w^{4} P(1 / w)=P(w)$, hence $A=E$ and $B=D$. It is easy to find that $P(0)=0$, hence $P(w)=B w^{3}+C w^{2}+B w$.

By Theorem 1(iv), $P(w)$ has a double root on $\partial U$, which must be equal to -1 , because of symmetry of $P(w)$ with respect to the real axis. Therefore (11) may be written in the form

$$
\frac{\zeta^{2} B(w+1)^{2} w^{\prime 2}}{w(w-b)^{2}(1-b w)^{2}}=-b_{1}^{2} .
$$

Passing in (12) to the limit as $\zeta \rightarrow 0$, we have

$$
B=-b_{1}^{2} b(1-b)^{2}<0 .
$$

Integrating now the equation (12), we see that $w=f(\zeta)$ is defined by

$$
\frac{(\sqrt{w}-\sqrt{b})(1+\sqrt{b} \sqrt{w})}{(\sqrt{w}+\sqrt{b})(1-\sqrt{b} \sqrt{w})}=\mu \zeta
$$

where $\mu \in \mathbb{R}$. Moreover, $|\mu|=1$ because the pre-image by $f$ of each point $w \in \partial U$ lies as well on $\partial U$. By passing in (13) to the limit as $\zeta \rightarrow 0$, we have $b_{1}=4 \mu b(1-b) /(1+b)$, which, in view of $b_{1}>0$, gives $\mu=1$. It is evident that the function which minimizes $b_{1}$ is the function (13) with $\mu=-1$ and then $b_{1}=-4 b(1-b) /(1+b)$. We have just proved

Theorem 2. If $f \in \mathcal{B}_{0}^{R}(b), 0<b<1$, then

$$
-\frac{4 b(1-b)}{1+b} \leq b_{1} \leq \frac{4 b(1-b)}{1+b}
$$

with equalities for the functions $w=f(\zeta)$ defined by (13) with $\mu= \pm 1$. The range of the function (13) is $U \backslash[-1,0]$.

The estimate (14) also follows from the one-quarter theorem and the fact that for $f \in \mathcal{B}_{0}^{R}(b)$ the function $F(z)=f(z) /(1 \pm f(z))^{2}$ is univalent and non-vanishing in $U$. However, it is instructive to see how this result follows from the variational method.

6. Estimation of $b_{2}=\frac{1}{2} f^{\prime \prime}(0)$ in $\mathcal{B}_{0}^{R}(b)$. Let now $\Psi(f)=b_{2}$. For the extremal function for this functional the equation (9) takes the form

$$
\frac{\zeta^{2} w^{\prime 2} P(w)}{w(b-w)^{3}(1-b w)^{3} b_{1}}=-2 b_{2}-b_{1}\left(\frac{1}{\zeta}+\zeta\right)
$$

where $P(w)=A w^{4}+B w^{3}+C w^{2}+B w+A$ with

(16) $A=-b^{3}\left(2 b_{2} b+b_{1}^{2}\right) b_{1}-b^{3}\left(3 b_{3}-b_{1}\right)\left(1-b^{2}\right)$,

(17) $B=\left(3 b_{2} b^{5}+6 b_{2} b^{3}-b_{2} b+3 b_{1}^{2} b^{2}\right) b_{1}+\left(3 b_{3}-b_{1}\right)\left(1-b^{2}\right) b\left(2 b^{3}+2 b\right)$,

$C=\left(-b_{2} b^{6}-9 b_{2} b^{4}-3 b_{2} b^{2}+b_{2}-6 b_{1}^{2} b\right) b_{1}$

$-\left(3 b_{3}-b_{1}\right)\left(1-b^{2}\right) b\left(b^{4}+4 b^{2}+1\right)$. 
In the case of maximum the right-hand side of (15) is non-positive on $\partial U$, in the case of minimum it is non-negative. It follows from Theorem 1(ii) that $b_{2} \geq 0$ in the case of maximum and $b_{2} \leq 0$ in the case of minimum. It can be assumed that $b_{1}>0$ in both cases. Indeed, if we take $f(-\zeta)$ instead of $f(\zeta)$ we can change the sign of $b_{1}$ without any change of $b_{2}$. Moreover, notice that, by Theorem $1(\mathrm{v}), w=-1$ must be a double root of the polynomial $P(w)$.

Two cases are possible: 1) $A=0,2) A \neq 0$.

In case 1$), P(w)=B w(w+1)^{2}$, the right-hand side of (15) must have a double root on $\partial U$ and this root is mapped by $f$ onto an end point of the interval $[-1, c]$, where $0 \leq c<b$. The right-hand side of (15) has a double root if and only if $b_{2}^{2}=b_{1}^{2}$ and hence $b_{2}=b_{1}$ in the case of maximum and $b_{2}=-b_{1}$ in the case of minimum. In the case of maximum the equation (15) has the form

$$
\frac{\zeta^{2} B(w+1)^{2} w^{\prime 2}}{b_{1}(b-w)^{3}(1-b w)^{3}}=-b_{1} \frac{(1+\zeta)^{2}}{\zeta},
$$

and in the case of minimum

$$
\frac{\zeta^{2} B(w+1)^{2} w^{\prime 2}}{b_{1}(b-w)^{3}(1-b w)^{3}}=-b_{1} \frac{(1-\zeta)^{2}}{\zeta} .
$$

We now show that a function for which $b_{2}$ attains its minimum cannot satisfy $\left(18^{\prime}\right)$. In fact, letting $\zeta \rightarrow 0$ in $(18)$ or $\left(18^{\prime}\right)$, we obtain

$$
B=\frac{b_{1}^{3}\left(1-b^{2}\right)^{3}}{(1+b)^{2}}>0 .
$$

Moreover, we easily conclude from (19), using the existence of an arc on $\partial U$ whose image through $w=f(\zeta)$ is an arc lying on $\partial U$, that the left-hand side of $\left(18^{\prime}\right)$ is non-positive on $\partial U$ while the right-hand side is non-negative there, which gives a contradiction.

Suppose that a function which maximizes $b_{2}$ satisfies (18). From $A=0$, (16), (17) and (19) we get

$$
b_{2}=b_{1}=\frac{1-b^{2}}{b+2} .
$$

On the other hand, according to (14), we have $\left|b_{1}\right| \leq 4 b(1-b) /(1+b)$. Therefore

$$
\frac{1-b^{2}}{b+2} \leq \frac{4 b(1-b)}{1+b}
$$

and so $-1+\frac{2}{3} \sqrt{3} \leq b<1$. Hence only for those $b$ the function maximizing $b_{2}$ can satisfy $(18)$. 
In case 2), two forms of equation (15) are possible:

or

$$
\frac{\zeta^{2} A(w+1)^{2}(w-1)^{2} w^{\prime 2}}{b_{1} w(b-w)^{3}(1-b w)^{3}}=-2 b_{2}-b_{1}\left(\frac{1}{\zeta}+\zeta\right)
$$

$$
\frac{\zeta^{2} A(w+1)^{2}(w-c)(w-1 / c)}{b_{1} w(b-w)^{3}(1-b w)^{3}}=-2 b_{2}-b_{1}\left(\frac{1}{\zeta}+\zeta\right)
$$

where $0<c<1$. If an extremal function satisfies $\left(21^{\prime}\right)$ it must map $U$ onto $U \backslash[-1,0]$, hence it is the function defined by (13) with $\mu=1$. But if the extremal function satisfies $(21)$, it can only minimize $b_{2}$, because the left-hand side of (21) is non-negative on $\partial U$, while it must be non-positive in the case of maximum. The right-hand side of (21) must have a double root on $\partial U$ and because $b_{2}<0$ in the case of minimum, we then have $b_{2}=-b_{1}$ and (21) has the form

$$
\frac{\zeta^{2} A(w+1)^{2}(w-1)^{2} w^{\prime 2}}{b_{1} w(b-w)^{3}(1-b w)^{3}}=-b_{1} \frac{(1-\zeta)^{2}}{\zeta} .
$$

Letting $\zeta \rightarrow 0$, we get

$$
A=b_{1}^{3} b\left(1-b^{2}\right),
$$

By (22), (23), (16) and (17) we have

$$
b_{2}=-b\left(1-b^{2}\right) .
$$

Summing up the above considerations, we see that a function maximizing $b_{2}$ in the case $-1+\frac{2}{3} \sqrt{3} \leq b<1$ can satisfy (18) or $\left(21^{\prime}\right)$, and in the case $0<b<-1+\frac{2}{3} \sqrt{3}$ it can only satisfy $\left(21^{\prime}\right)$, and then it must be the function (13) with $\mu=1$. But if a function realizes minimum of $b_{2}$, it can satisfy (22) or $\left(21^{\prime}\right)$. The coefficient $b_{2}$ for the function (13) with $\mu=1$ is given by

$$
b_{2}=\frac{-8 b(1-b)}{(1+b)^{3}}\left(b^{2}+2 b-1\right)
$$

therefore the function (13) can maximize $b_{2}$ only for $0<b \leq-1+\sqrt{2}$, and can minimize it only for $-1+\sqrt{2} \leq b<1$. It is easy to verify that for each $0<b<1$

$$
-b\left(1-b^{2}\right)<\frac{-8 b(1-b)}{(1+b)^{3}}\left(b^{2}+2 b-1\right) .
$$

We conclude that the function minimizing $b_{2}$ is not the function (13), therefore it must satisfy equation (22), so that

$$
\min _{\mathcal{B}_{0}^{R}(b)} b_{2}=-b\left(1-b^{2}\right) \text {. }
$$

Concerning a function maximizing $b_{2}$, for $0<b \leq-1+\frac{2}{3} \sqrt{3}$ it satisfies $\left(21^{\prime}\right)$, therefore it is the function (13). In the case $-1+\sqrt{2} \leq b<1$ a 
maximizing function must satisfy (18). Only for $-1+\frac{2}{3} \sqrt{3} \leq b<-1+\sqrt{2}$ it is necessary to compare $b_{2}$ from formula (20) with $b_{2}$ from (24). It turns out that for $-1+\frac{2}{3} \sqrt{b} \leq b \leq-1+\sqrt{2}$,

$$
\frac{-8 b(1-b)\left(b^{2}+2 b-1\right)}{(1+b)^{3}} \leq \frac{1-b^{2}}{b+2},
$$

with equality only for $b=-1+\frac{2}{3} \sqrt{3}$, so for $b \in\left[-1+\frac{2}{3} \sqrt{3},-1+\sqrt{2}\right]$ a function maximizing $b_{2}$ satisfies (18).

Equation (18) can be integrated and its solution $w=f(\zeta)$ with the initial condition $f(0)=b$ satisfies

$$
\frac{(1+b)^{2}(w-1)^{2}}{(b+2)(w-b)(1-b w)}=\zeta+\frac{1}{\zeta}-2 .
$$

The function (25) maps $U$ onto $U \backslash[-1, c]$, where

$$
c=\frac{2 b^{3}+3 b^{2}+3-2\left(1-b^{2}\right) \sqrt{(b+2)(b+1)}}{3 b^{2}+6 b-1}
$$

for $b \in\left(-1+\frac{2}{3} \sqrt{3}, 1\right)$ and $c=0$ for $b=-1+\frac{2}{3} \sqrt{3}$.

In this way we have established the following theorem.

THEOREM 4. If $f \in \mathcal{B}_{0}^{R}(b)$, then

$$
-b\left(1-b^{2}\right) \leq b_{2} \leq \begin{cases}\frac{-8 b(1-b)\left(b^{2}+2 b-1\right)}{(1+b)^{3}}, & 0<b \leq-1+\frac{2}{3} \sqrt{3}, \\ \frac{1-b^{2}}{b+2}, & -1+\frac{2}{3} \sqrt{3} \leq b<1 .\end{cases}
$$

These estimates are sharp. The minimum is realized by a function which satisfies (21), maximum in $\left(0,-1+\frac{2}{3} \sqrt{3}\right]$ by the function (13) with $\mu=1$, and in $\left[-1+\frac{2}{3} \sqrt{3}, 1\right)$ by a function which satisfies (18).

Prokhorov and Szynal [5] obtained the exact bound on $\left|b_{2}\right|$ in the class $\mathcal{B}_{0}(b)$, using the connections between $\mathcal{B}_{0}(b)$ and the class of Gel'fer and Bieberbach-Eilenberg functions and, in particular, an estimate of $\left|b_{2}\right|$ for this last class.

\section{References}

[1] G. M. Goluzin, Geometric Theory of a Complex Variable, Transl. Math. Monographs 26, Amer. Math. Soc., Providence, R.I., 1969.

[2] J. A. Hummel and B. Pinchuk, Variations for bounded nonvanishing univalent functions, J. Anal. Math. 44 (1984-85), 183-199.

[3] J. A. Hummel and M. M. Schiffer, Variational methods for Bieberbach-Eilenberg functions and for pairs, Ann. Acad. Sci. Fenn. Ser. AI Math. 3 (1977), 3-42.

[4] C. Pommerenke, Univalent Functions, Göttingen, 1975. 
[5] D. V. Prokhorov and J. Szynal, Coefficient estimates for bounded nonvanishing functions, Bull. Acad. Polon. Sci. Sér. Sci. Math. 29 (1981), 223-230.

[6] J. Śladkowska, Sur une famille des fonctions univalentes et bornées, Demonstratio Math. 22 (1989), 973-982.

[7] —, Sur la famille des fonctions univalentes, bornées et symétriques qui n'atteignent pas une valeur fixée, Ann. Polon. Math. 52 (1990), 147-160.

[8] -, A variational method for univalent nonvanishing functions in the unit disk, Folia Sci. Univ. Tech. Resov. 129, Math. 16 (1994), 63-77.

Institute of Mathematics

Silesian Technical University

Kaszubska 23

44-100 Gliwice, Poland 